University of Nebraska - Lincoln

DigitalCommons@University of Nebraska - Lincoln

$1-23-2008$

\title{
Polyelectrolyte mediated scalable synthesis of highly stable silver nanocubes in less than a minute using microwave irradiation
}

\author{
Subrata Kundu \\ University of Nebraska - Lincoln \\ Vivek C. Maheshwari \\ University of Nebraska-Lincoln, vmaheshw@uwaterloo.ca \\ Sanjun Niu \\ University of Nebraska - Lincoln \\ Ravi F. Saraf \\ University of Nebraska-Lincoln, rsaraf2@unl.edu
}

Follow this and additional works at: https://digitalcommons.unl.edu/cbmesaraf

Part of the Biomechanics and Biotransport Commons

Kundu, Subrata; Maheshwari, Vivek C.; Niu, Sanjun; and Saraf, Ravi F., "Polyelectrolyte mediated scalable synthesis of highly stable silver nanocubes in less than a minute using microwave irradiation" (2008). Ravi Saraf Publications. 9.

https://digitalcommons.unl.edu/cbmesaraf/9

This Article is brought to you for free and open access by the Chemical and Biomolecular Research Papers -- Faculty Authors Series at DigitalCommons@University of Nebraska - Lincoln. It has been accepted for inclusion in Ravi Saraf Publications by an authorized administrator of DigitalCommons@University of Nebraska - Lincoln. 


\title{
Polyelectrolyte mediated scalable synthesis of highly stable silver nanocubes in less than a minute using microwave irradiation
}

\author{
Subrata Kundu, Vivek Maheshwari, Sanjun Niu, and Ravi F. Saraf \\ Department of Chemical and Biomolecular Engineering, University of Nebraska-Lincoln, NE 68588-0643, USA \\ Corresponding author email: rsaraf@unlnotes.unl.edu
}

\begin{abstract}
Silver nanocubes were synthesized for the first time in large quantities on gold seed in the presence of a polyelectrolyte using microwave heating for 60-120 s. Our experiment indicates that the polyelectrolyte directs the growth of the particle in a specified crystallographic direction resulting in the faceted particle, i.e. a nanocube. The nanocubes are stable for at least 2 months in ambient conditions.
\end{abstract}

\section{Introduction}

The possibility of controlling the shape of nanoparticles to more complex geometries such as triangles, rods and cubes provides another handle (other than size) to manipulate properties of nanomaterials. Shape-control synthesis of inorganic nanostructures has received considerable attention in recent years because of applications in catalysis [1], optics [2], microelectronics [3], magnetics [4] and medical diagnostics [5]. A variety of chemical methods have been developed for the synthesis of metal nanostructures that have well-controlled shapes, such as wires [6-8], rods [9-11], disks [12], cubes $[13,14]$, belts [15], plates [16], prisms [17] and branched multipods [18]. Nanocubes can provide properties different from spherical nanoparticles, for example pinning of the magnetic domains to attain ferromagnetic properties at the nanoscale [19], and self-assembly into highly packed structures that may be used as templates to make superlattices for high density storage applications [20]. Recently, we have synthesized gold nanocubes [14] with cetyltrimethylammonium bromide (CTAB) using UV irradiation. Composite nanocubes of $\mathrm{Au}$ on Ag may lead to highly conducting noble nanomaterials that are easier to pack than spherical particles [13]. Well formed, monodispersed nanocubes of silver have been synthesized from $\mathrm{AgNO}_{3}$ in ethylene glycol [13] and in water using a modified silver mirror reaction [21]. Typically, current nanocube synthesis methods have a long processing time, requir- ing a high temperature with multiple steps $[13,21]$. Recently, microwaves (MWs) as a heat source have been used to synthesize spherical nanoparticles of metals like $\mathrm{Ag}$ [22], $\mathrm{Au} / \mathrm{Pd}$ [23] and semiconductor rods and wires [24] at a significantly higher speed compared to conventional thermal convection. In this communication we describe a process using MWs to synthesize silver nanocubes in a reaction time of $<2$ min using polymers to guide the growth process. The key discovery was "guiding" the synthesis of the particles with a polyelectrolyte rather than conventional low molecular weight amphiphiles that do not yield nanocubes, and the method is straightforward, cost-effective and scalable.

\section{Experimental details}

\subsection{Reagents}

Poly(styrene sulfonate), (PSS) and poly-allylamine hydrochloride (PAH), each of average molecular weight $7 \times 10^{4}$ $\mathrm{Da}$, were purchased from Scientific Polymer Products and Aldrich, respectively. Silver nitrate $(99.99 \%)$, hydrogen tetrachloroaurate trihydrate $\left(\mathrm{HAuCl}_{4} \cdot 3 \mathrm{H}_{2} \mathrm{O}, 99.9 \%\right)$ and sodium borohydride $\left(\mathrm{NaBH}_{4}\right)$ were purchased from Sigma-Aldrich. CTAB and sodium dodecyl sulfate (SDS) were purchased from Sigma. Tri-sodium citrate dihydrate $\left(\mathrm{Na}_{3} \mathrm{C}_{6} \mathrm{H}_{5} \mathrm{O}_{7} \cdot 2 \mathrm{H}_{2} \mathrm{O}\right)$ was purchased from Aldrich. Ultrapure distilled (UPD) water was used for the synthesis. 


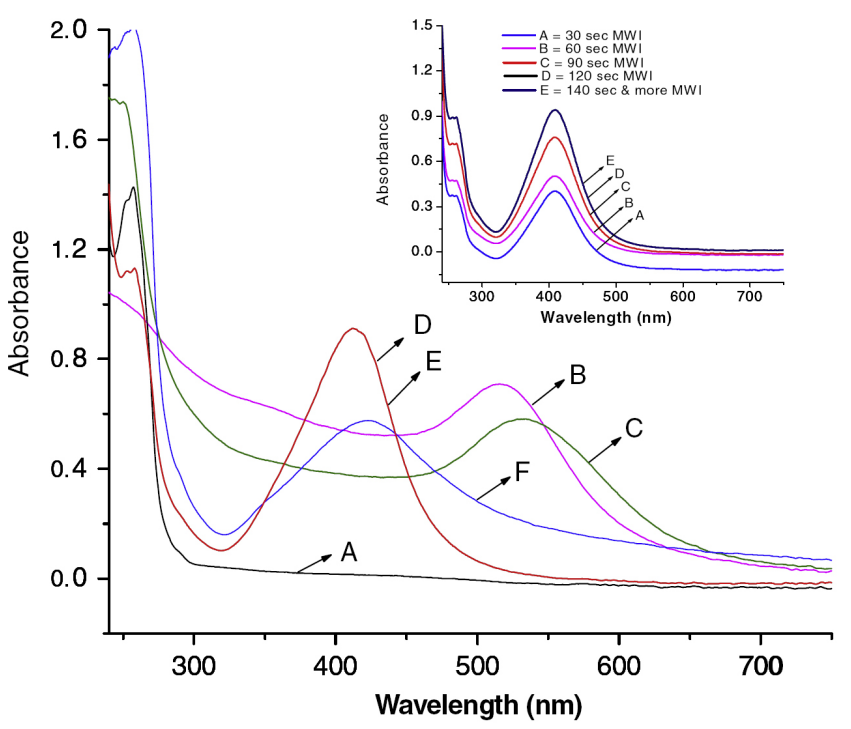

Figure 1. UV-visible spectra at various stages of silver nanocube synthesis. A) absorption spectra of PSS ( $0.1 \%$ by weight) in water; B) surface plasmon resonance band for gold seed particles; C) spectrum of the mixture of PSS, gold seed and silver nitrate before MW irradiation; D) surface plasmon band of silver cubes in PSS; E) surface plasmon band of the same solution after 2 months of aging in air; and F) surface plasmon band of silver particles using SDS instead of PSS in the presence of $\mathrm{Au}$ seeds. The inset shows the SPR absorption with increasing MW exposure.

\subsection{Synthesis of gold seed particles}

A $20 \mathrm{ml}$ aqueous solution containing $2.5 \times 10^{-4} \mathrm{M} \mathrm{HAuCl}_{4}$ and $2.5 \times 10^{-4} \mathrm{M}$ tri-sodium citrate was prepared in a flask. Next, $0.6 \mathrm{ml}$ of ice-cold $0.1 \mathrm{M} \mathrm{NaBH}_{4}$ solution was added to the solution all at once while stirring. The solution turned pink immediately after adding $\mathrm{NaBH}_{4}$, indicating particle formation. The particles in this solution were used as seeds within $2-5 \mathrm{~h}$ after preparation. The average particle size measured from a transmission electron micrograph was $4 \pm 0.7 \mathrm{~nm}$ (see TEM image in figure 4(A)). The citrate serves only as a capping agent since it cannot reduce gold salt at room temperature $\left(25^{\circ} \mathrm{C}\right)$.

\subsection{General route for the synthesis of silver nanocubes on PSS by MW heating}

PSS solution ( $0.1 \%$ by weight) was prepared by dissolving it in UPD water and stirring over $10 \mathrm{~h}$. A stock solution of $0.1 \mathrm{M}$ aqueous silver nitrate $\left(\mathrm{AgNO}_{3}\right)$ was made and kept in the dark for protection against light. In a typical synthesis, $4 \mathrm{ml}$ of PSS $(0.1 \%)$ was mixed with $200 \mu \mathrm{l}$ of $(0.1 \mathrm{M})$ silver nitrate solution and the mixture stirred for 2-3 min using a magnetic stirrer. $50 \mu \mathrm{l}$ of freshly prepared gold seed solution was added to it and allowed to equilibrate for $\sim 2 \mathrm{~min}$. The equilibrium was determined by the stability of the surface plasmon resonance (SPR) band. The resulting solution was heated by MWs for $30-120 \mathrm{~s}$ with an intermittent pause after every $10 \mathrm{~s}$ to cool the reaction vessel. The silver particle formation started after just $20 \mathrm{~s}$ of MW heating as observed by UV-visible spectrom- etry. The formation of silver nanoparticles was evident by the appearance of a light yellowish coloration of the solution. After complete formation the solution became deep yellowish in color and the color remained stable for at least 2 months of storage in the ambient environment in the dark without any change in optical properties.

\subsection{Instruments and preparation of sample for FESEM analysis}

UV-visible absorption spectra were recorded in an OceanOptics absorbance spectrophotometer equipped with a $1 \mathrm{~cm}$ quartz cuvette holder for liquid samples. Field emission scanning electron microscope (FESEM) analysis was performed using a Hitachi S-4700. TEM analysis was performed using a Hitachi-H-9000 NAR. The samples for TEM were prepared by placing a drop of fresh silver nanocube solution on a carbon film-coated copper grid followed by slow evaporation of solvent at ambient condition. A domestic MW oven (Sanyo Company, EM-Z200S, $1000 \mathrm{~W}, 60 \mathrm{~Hz}$ ) was used for MW irradiation for nanocube synthesis.

FESEM studies were performed on a Si chip with a native oxide layer. The chip was cleaned thoroughly with ethanol and Piranha $\left(30 \% \mathrm{H}_{2} \mathrm{O}_{2}\right.$ and $\left.70 \% \mathrm{H}_{2} \mathrm{SO}_{4}\right)$ followed by final cleaning with ethanol and acetone. The chip was then air dried and dipped in a $0.1 \%$ solution of poly-allylamine hydrochloride (PAH) overnight, followed by a vigorous wash to remove excess PAH leaving a monolayer. The sample was dried, and placed in silver nanoparticle solution for $30 \mathrm{~min}$ to deposit Ag nanocubes.

\section{Results and discussion}

Silver nanocubes are synthesized in a $1000 \mathrm{~W}$ MW oven in the presence of Au seed (figure 4(A)) using PSS as a stabilizing agent. The key discovery was "guiding" the synthesis of the particles with a polyelectrolyte rather than conventional low molecular weight amphiphiles that do not yield nanocubes. PSS tends to support the growth of Ag particle in certain specific crystallographic directions leading to shape control. The yield of cubes is high, with very few spherical or other shaped particles.

Figure 1 compares the UV-visible spectrum of the solution at various stages of the process. The PSS solution exhibits the expected UV absorption at $\sim 265 \mathrm{~nm}$ due to the benzene rings (curve A), and the Au seed solution shows the SPR absorption band at $\sim 515 \mathrm{~nm}$ (curve B). The UV spectrum of the PSS, $\mathrm{Au}$ seed and $\mathrm{AgNO}_{3}$ solution (before MW exposure) is a superposition of the PSS and Au seed spectrum (curve C). However, the SPR band of the Au seed is broadened and redshifted. The red-shift is attributed to deposition of the negatively charged Au nanoparticle on a Ag-PSS complex causing an increase in apparent diameter. Only $\sim 1-2 \mathrm{~min}$ is required to obtain the stable, modified SPR band, indicating that the modification of gold seed is rapid. After $120 \mathrm{~s}$ of exposure to MWs, 

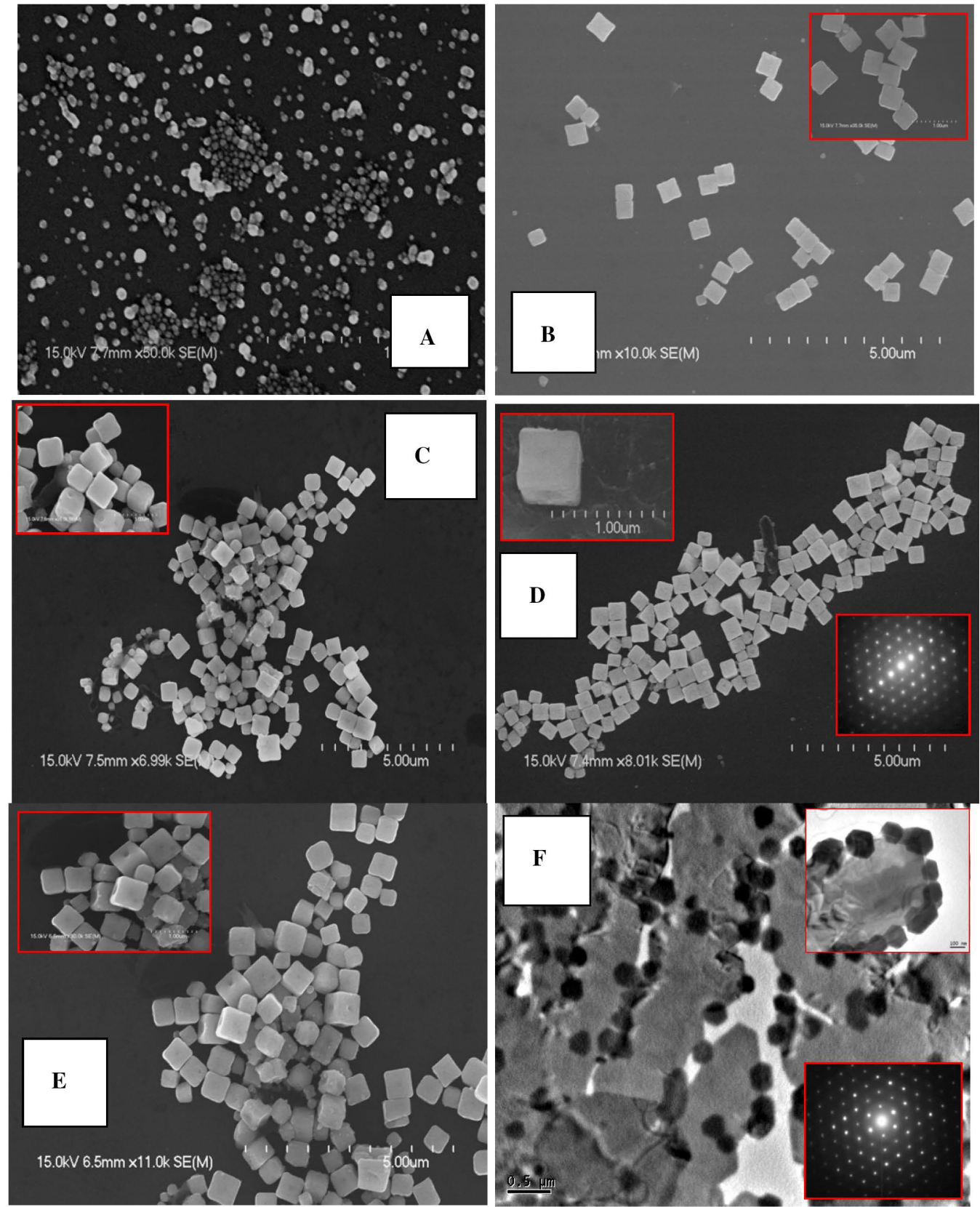

Figure 2. FESEM and TEM images of silver nanoparticles under different conditions. (A) FESEM image of silver nanoparticles after $30 \mathrm{~s}$ of MW exposure; (B), (C) and (D) FESEM images of silver nanocubes after 60, 90 and 120 s exposure of MW, respectively; (E) image of the cubes made by 120 s MW exposure after 2 months of aging in air; and (F) TEM image of hexagonal single-crystal nanoparticles of silver in PSS, without the Au seed. The electron diffraction pattern obtained by TEM in (D) and (F) shows that the silver particles are single crystals.

the Au plasmon peak disappears and a strong SPR peak for Ag appears at $415 \mathrm{~nm}$ (curve D). The SPR band is consistent with the formation of Ag nanoparticles [21, 25] (figures 2(B)(D)). We do not observe two peaks as reported by Xia's group [13] but a single peak identical with that of silver nanoparticles (with cubic shape) prepared chemically by the reduction of silver ions [21]. The solution is remarkably stable with no sign of oxidation or sulfide formation for at least 2 months of storage in an ambient environment (curve $\mathrm{E}$, which is coincident on curve D). The SPR absorption for silver monotonically increases with MW exposure and saturates at $120 \mathrm{~s}$, indicating completion of the reaction (inset of figure 1).
Replacing the PSS with its monomer (styrene sulfonate) or a negatively charged surfactant such as SDS yields spherical nanoparticles (curve F), but no cubes are formed (figure $3)$. The significantly broader and red-shifted SPR band indicates that the particles are large with a broad size distribution.

The structure of the particles is observed by FESEM of solution-dried particles on a Si substrate. For MW exposure times of less than $60 \mathrm{~s}$ particles are spherical (figure 2(A)). For exposure times of $60 \mathrm{~s}$ and more the particle are cubic (figures 2(B)-(D)). The FESEM images suggest that the cubes tend to self-assemble along their sides. The yield of cubes is best at $120 \mathrm{~s}$. The side of the cube (taken over an average of 40 cube) 

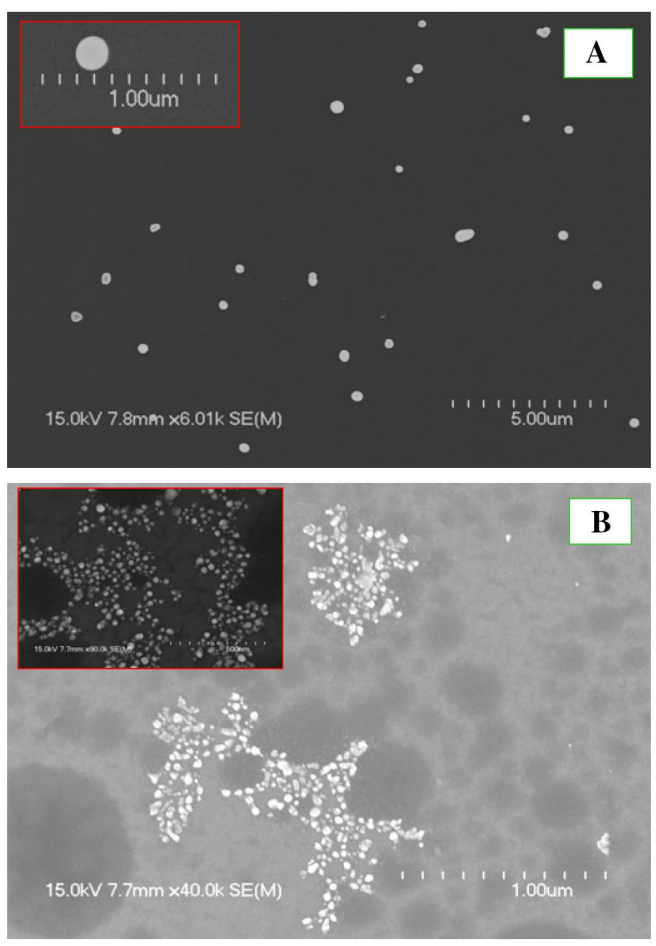

Figure 3. FESEM image of silver nanoparticles synthesized in (A) SDS and (B) PSS monomer under MW exposure for $120 \mathrm{~s}$. The inset shows their corresponding high magnification images.

for 60,90 and $120 \mathrm{~s}$ exposure time is $450 \pm 5,480 \pm 7$ and 490 $\pm 10 \mathrm{~nm}$, respectively.

In the proposed method we have varied the concentration of PSS, silver salt and gold seed and the microwave irradiation time. The best results (uniform nanocubes) are obtained with the conditions given in the experimental section. No cube formation is observed when the PSS concentration is increased from $0.1 \%$ to $1 \%$ or decreased from $0.1 \%$ to $0.01 \%$ within $120 \mathrm{~s}$ MW irradiation. Similarly silver cubes are only formed at a higher concentration $(0.1 \mathrm{M})$ of $\mathrm{AgNO}_{3}$; no cubes formed at a lower concentration $(0.01 \mathrm{M})$. The polydispersity and presence of other shapes with a cubic structure is perhaps due to nonuniform conditions during the reaction. To obtain a more uniform structure, a better MW cavity with uniform intensity is required. Although the shape and size of the particles change significantly from 30 to $120 \mathrm{~s}$ exposure, the position of the plasmon peak is unchanged. The invariance is attributed to the characteristic radius of curvature of the cubes' rounded edges. Consistent with the UV-visible spectrum (curves D and E) the morphology of the cubes is stable over 2 months (compare figures 2(D) and (E)).

The role of MW and PSS is central to the synthesis of nanocubes. Heating $\mathrm{AgNO}_{3}$ salt solution by a resistor-heater or in a MW oven in the presence (or absence) of Au seed causes thermal reduction of $\mathrm{Ag}^{+} \rightarrow \mathrm{Ag}^{0}$ [26]. Thus, microwaves are essentially a heat source for rapid and uniform heating. However, without the PSS the precipitate is clusters of microparticles with no evidence of nanoparticles (of any shape, see figure 4(B)).

In the presence of PSS, without the Au seed, well defined hexagonal single-crystal nanoparticles are formed at the polymer/bulk-solution interface (figure 2(F)). The faceted growth of particles $<300 \mathrm{~nm}$, that is not observed with other capping agents such as negatively charged SDS (figure 3(A)), indicates that PSS promotes growth in certain selective crystallographic axes. Such selectivity is conjectured in the synthesis of Au nanorods using CTAB [27]. We note in passing that the use of positively charged capping compounds such as CTAB or positively charged polyelectrolyte (i.e. PAH) is not possible because the $\mathrm{Ag}^{+}$reacts with the counter-anion of the surfactant to readily form a silver salt precipitate. Another role of PSS is as a "reservoir" of $\mathrm{Ag}^{+}$ions. Prior to MW exposure we first allow equilibrium between the PSS and $\mathrm{AgNO}_{3}$ in solution. In this step the $\mathrm{Ag}^{+}$ions and the $-\left(\mathrm{SO}_{3}\right)^{-}$of PSS form a PSS-Ag salt. On MW exposure, as the nucleation and growth occurs at the polymer/electrolyte solution interface, the ions are supplied from the polymer side because of higher concentration than in aqueous solution. Further, as the $\mathrm{Ag}^{+}$ions are deposited on the growing nuclei they are replenished from the solution. Such a crystal growth process partially supports the PSS-mediated faceted growth hypothesis at the interface, and is consistent with the fact that for SDS, which can only have
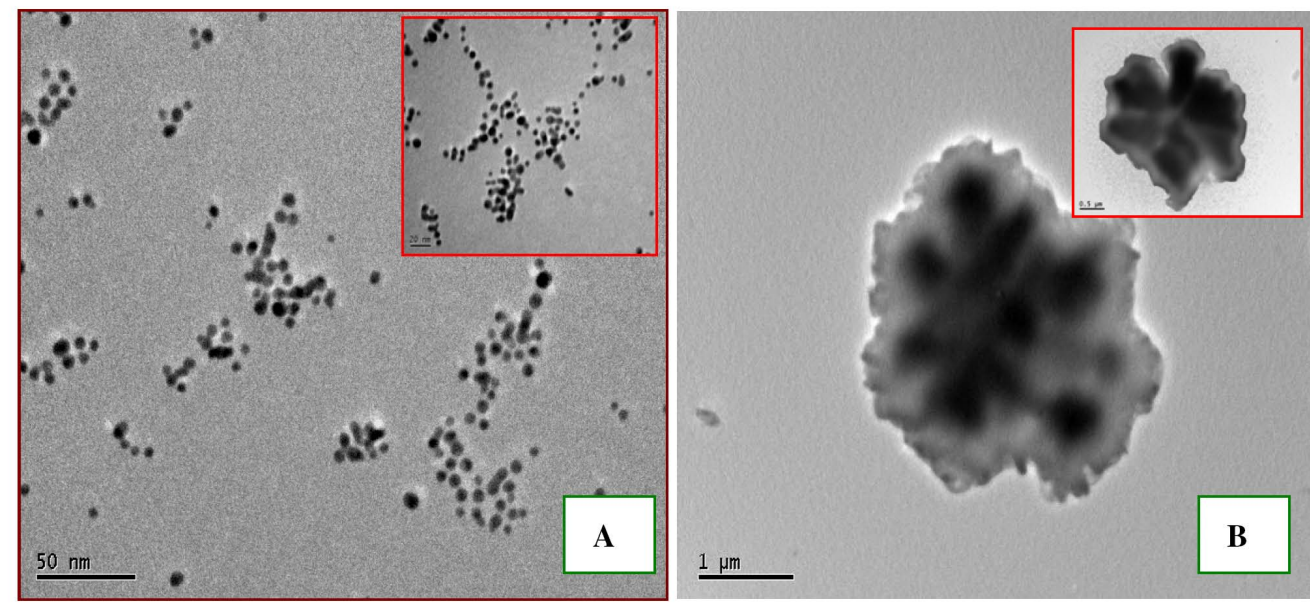

Figure 4. (A) Low and high magnification TEM images of gold seed particles showing an average particle size of $\sim 4 \pm 0.7 \mathrm{~nm}$. (B)Low and high magnification TEM images of silver microparticles synthesized with gold seeds in the absence of PSS, under MW exposure for $120 \mathrm{~s}$. 
one ion per molecule (in contrast to over 200 ions per PSS molecule), the growth is much slower with no facets (compare figures 2(D) and 3(A)).

The fairly uniform size distribution of the hexagonal crystal habit (figure $2(\mathrm{~F})$ ) also indicates that the nucleation is heterogeneous. On addition of Au seed to PSS-Ag salt solution (i.e. figures 2(A)-(E)), the SPR peak of Au seed redshifts, indicating absorption on the seed, to cause an increase in apparent size (curves $\mathrm{B}$ and $\mathrm{C}$ in figure 1). If the deposition was of just the free $\mathrm{Ag}^{+}$ions in the solution, the resulting particles on MW exposure would be microparticles as mentioned in the above paragraph.

The production of a faceted structure implies that the absorbing species must be the PSS-Ag salt. However, as the Au seed is negatively charged, the absorption may be mediated by $\mathrm{Ag}^{+}$ions. On heating such a structure with $\mathrm{MW}$, the $\mathrm{Ag}^{+}$ions will deposit on the Au seed with growth in the preferred crystallographic direction guided by PSS, to form the observed faceted structure, i.e. the cubes. Such a directed nucleation has been suggested for the synthesis of nanocubes [14, 21]. In both reports and our case the mechanism at the atomic scale is not fully understood.

\section{Conclusions}

In summary, we demonstrate that silver nanocubes can be synthesized in 60-120 s using MWs as the heat source and PSS to control the shape of the particle. The proposed method is very straightforward, time-efficient and scalable. We conjecture that the role of PSS is to direct the growth along specific crystallographic axes and act as a "reservoir" for $\mathrm{Ag}^{+}$supply. Utilizing multiple ions, this approach may lead to a quick manufacturing method to synthesize nanocubes of complex composite structures for application in catalysis and nanoelectronics.

\section{Acknowledgments}

Financial support from NSF (NER-0608877) is appreciated. The authors acknowledge Dr X. Z. Li at the electron microscopy center in the University of Nebraska-Lincoln for help with HR-TEM.

\section{References}

[1] Crooks R M, Zhao M, Sun L, Chechik V, and Yeung L K 2002 Acc. Chem. Res. 34181

[2] Taleb A, Petit C, and Pileni M P 1998 J. Phys. Chem. B 1022214

[3] Klaus T, Joerger R, Olsson E, and Granqvist C-G 1999 Proc. Natl Acad. Sci. 9613611

[4] Sun S, Murray C B, Weller D, Folks L, and Moser A 2000 Science 2871989

[5] Haes A J and Van Duyne R P 2002 J. Am. Chem. Soc. 12410596

[6] Sun Y, Mayers B, Herricks T, and Xia Y 2003 Nano Lett. 3955

[7] Sun Y, Yin Y, Mayers B, Herricks T, and Xia Y 2002 Chem. Mater. 144736

[8] Sun Y, Mayers B, and Xia Y 2003 Adv. Mater. 15641

[9] Jana N R, Gearheart L, and Murphy C J 2001 J. Phys. Chem. B 1054065

[10] Murphy C J and Jana N R 2002 Adv. Mater. 1480

[11] Kim F, Song J H, and Yang P 2002 J. Am. Chem. Soc. 124 14316

[12] Hao E C, Kelly K L, Hupp J T, and Schatz G C 2002 J. Am. Chem. Soc. 12415182

[13] Sun Y and Xia Y 2002 Science 2982176

[14] Kundu S, Panigrahi S, Praharaj S, Basu S, Ghosh S K, Pal A, and Pal T 2007 Nanotechnology 18075712

[15] Sun Y, Mayers B, and Xia Y 2003 Nano Lett. 5675

[16] Sun Y and Xia Y 2003 Adv. Mater. 15695

[17] Jin R, Cao Y, Mirkin C A, Kelly K L, Schatz G C, and Zheng J G 2001 Science 2941901

[18] Hao E, Bailey R C, Schartz G C, Hupp J T, and Li S 2004 Nano Lett. 4327

[19] Song Y-Y, Zhang K, and Xia X-H 2006 Appl. Phys. Lett. 88 053112

[20] Dumestre F, Chaudret B, Amiens C, Renaud P, and Fejes P 2004 Science 303821

[21] Yu D and Yam V W 2004 J. Am. Chem. Soc. 12613200

[22] Pastoriza-Santos I and Liz-Marzán L 2002 Langmuir 182888

[23] Harpeness R and Gedanken A 2004 Langmuir 203431

[24] Panda A B, Glaspell G P, and El-Shall M S 2006 J. Am. Chem. Soc. 1282790

[25] Pal T, Sau T K, and Jana N R 1997 Langmuir 131481

[26] Pol V G, Srivastava D N, Palchik O, Palchik P, Slifkin M A, Weiss A M, and Gedanken A 2002 Langmuir 183352

[27] Petroski J M, Wang Z L, Green T C, and El-Sayed M A 1998 J. Phys. Chem. B 1023316 